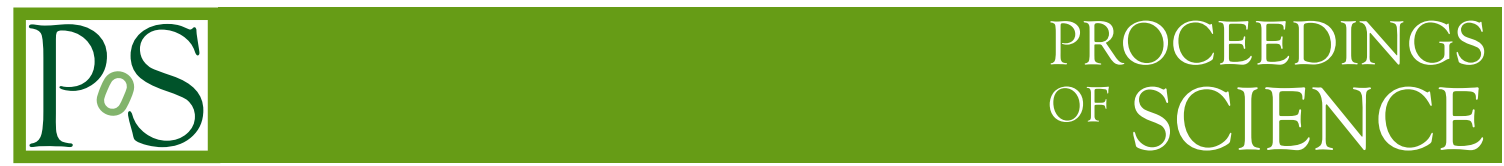

\title{
Extractions of the QCD coupling in ATLAS
}

\author{
Fernando Barreiro (On behalf of the ATLAS Collaboration)* \\ Facultad de Ciencias, Universidad Autónoma de Madrid, 28049 Madrid \\ E-mail: fernando.barreiro@uam.es
}

\begin{abstract}
I discuss two recent measurements by the ATLAS collaboration [1] at the LHC on transverse energy-energy correlations and on the transverse momentum and rapidity dependence of dijet azimuthal decorrelations at $\sqrt{s}=8 \mathrm{TeV}$. They are based on the 2012 sample with an integrated luminosity of $20.2 \mathrm{fb}^{-1}$. They are used to determine the strong coupling constant and to probe its running up to scales of order $2 \mathrm{TeV}$.
\end{abstract}

$\alpha_{s}$ (2019): Workshop on precision measurements of the QCD coupling constant 11-15 February, 2019

Trento, Italy

${ }^{*}$ Speaker. 


\section{Measurement of transverse energy-energy correlations}

Transverse energy-energy correlations (TEEC) are defined as the weighted average of azimuthal differences between jet pairs [2] i.e.:

$$
\frac{1}{\sigma} \frac{d \Sigma}{d(\cos \phi)}=\frac{1}{\sigma} \sum_{i j} \int \frac{d \sigma}{d x_{\mathrm{Ti}} d x_{\mathrm{Tj}} d(\cos \phi)} x_{\mathrm{Ti}} x_{\mathrm{Tj}} d x_{\mathrm{Ti}} d x_{\mathrm{Tj}}
$$

where the sum runs over all pairs of jets in the final state with azimuthal angular difference $\phi$ and $x_{\mathrm{Ti}}=\frac{E_{\mathrm{Ti}}}{E_{\mathrm{T}}}$ is the transverse energy carried by jet $i$ in units of the sum of jet transverse energies $E_{\mathrm{T}}=\sum_{i} E_{\mathrm{Ti}}$.

In order to cancel uncertainties which are constant over $\cos \phi \in[-1,1]$, it is useful to define the azimuthal asymmetry of the TEEC (ATEEC) as

$$
\left.\frac{1}{\sigma} \frac{d \Sigma^{\text {asym }}}{d(\cos \phi)} \equiv \frac{1}{\sigma} \frac{d \Sigma}{d(\cos \phi)}\right|_{\phi}-\left.\frac{1}{\sigma} \frac{d \Sigma}{d(\cos \phi)}\right|_{\pi-\phi} .
$$

Next to leading order (NLO) corrections have been recently calculated [3] using the NLOJET++ code [4]. They have been found to be moderate, with PDF uncertainties also well under control, thus making this observable suitable for a precise test of pQCD and for a determination of the strong coupling constant. Recent measurements of the TEEC have been published by the ATLAS collaboration at $8 \mathrm{TeV}$ [5]. The analysis represents an extension of previous measurements at $7 \mathrm{TeV}$ [6]. The selection criteria require at least two anti- $k_{\mathrm{T}}$ jets $(\mathrm{R}=0.4)$ such that $H_{\mathrm{T} 2}=p_{\mathrm{T} 1}+p_{\mathrm{T} 2} \geq 800 \mathrm{GeV}$ with the transverse momenta of any additional jet above $100 \mathrm{GeV}$. To ensure that jet reconstruction is optimal, jets are required to be central with $\left|\eta_{\text {jet }}\right| \leq 2.5$. In the 2012 data sample used in this analysis the total number of selected events is $6.2 \times 10^{6}$. The data is further binned in six intervals in $H_{\mathrm{T} 2}$, the first one between $800-850 \mathrm{GeV}$ and the last one above $1400 \mathrm{GeV}$. The data are corrected for detector effects using either a bin-by-bin correction or a Bayesian unfolding. The systematic uncertainties are dominated by the choice of the MC model used in the unfolding, modelling, and by the jet energy scale, JES, which include 67 independent sources. The total systematic uncertainty for the TEEC measurements are always below the 4-5\% level.

The unfolded data is fitted to NLOJET++ predictions which are dependent on $\alpha_{\mathrm{s}}\left(m_{\mathrm{Z}}\right)$. The choice of renormalization and factorization scales is $\mu_{\mathrm{R}}=H_{\mathrm{T} 2} / 2$ with $\mu_{\mathrm{F}}=\mu_{\mathrm{R}} / 2$. The theoretical uncertainties are dominated by the scale uncertainties, while those due to the PDF eigenvectors are subdominant. The comparison between unfolded data for the TEEC (ATEEC) and theoretical predictions is fair as illustrated in Fig. 1 for the highest bin in $H_{\mathrm{T} 2}$ namely $H_{\mathrm{T} 2} \geq 1400 \mathrm{GeV}$.

The values for $\alpha_{\mathrm{s}}$ obtained at scales $H_{\mathrm{T}} / 2$ from the TEEC and its asymmetry are shown in Fig. 2. They are in very good agreement with the renormalization group equation (RGE) dependence predicted in QCD. In fact, the goodness of this agreement has been used recently to put limits on new coloured fermions in a way which is independent of assumptions about their decay modes [7].

From a global fit to the complete data sample the following values for the strong coupling constant at the $Z$ boson mass are obtained:

$$
\begin{aligned}
& \alpha_{\mathrm{s}}^{\mathrm{TEEC}}\left(m_{\mathrm{Z}}\right)=0.1162 \pm 0.0011(\exp )_{-0.0061}^{+0.0076}(\text { scale }) \pm 0.0018(\mathrm{PDF}) \pm 0.0003(\mathrm{NP}) \\
& \alpha_{\mathrm{s}}^{\mathrm{ATEEC}}\left(m_{\mathrm{Z}}\right)=0.1196 \pm 0.0013(\exp )_{-0.0013}^{+0.0061}(\text { scale }) \pm 0.0017(\mathrm{PDF}) \pm 0.0004(\mathrm{NP})
\end{aligned}
$$



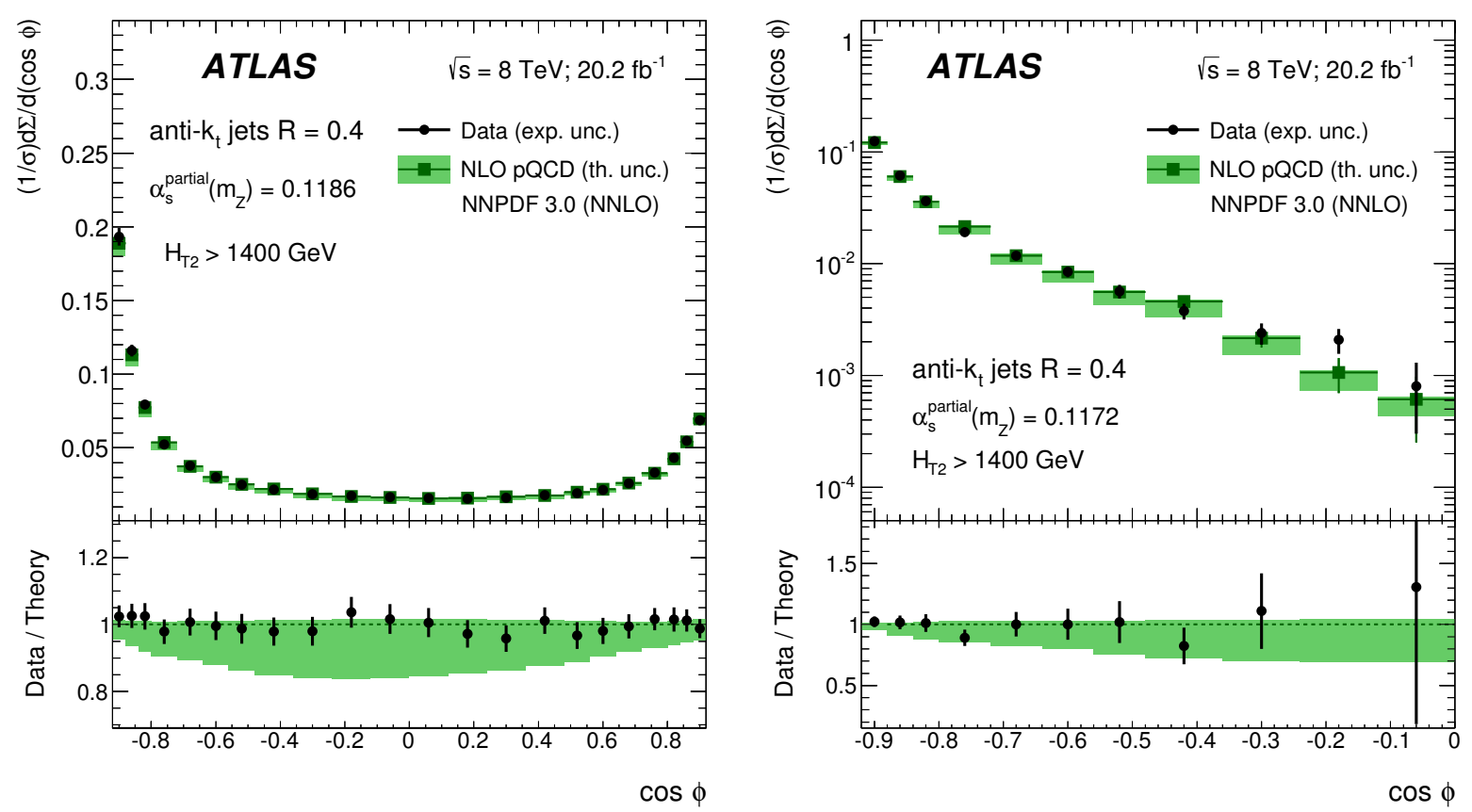

Figure 1: Results of fitting the unfolded data on the TEEC (left) and its asymmetry (right) to NLOJET++ predictions [5].
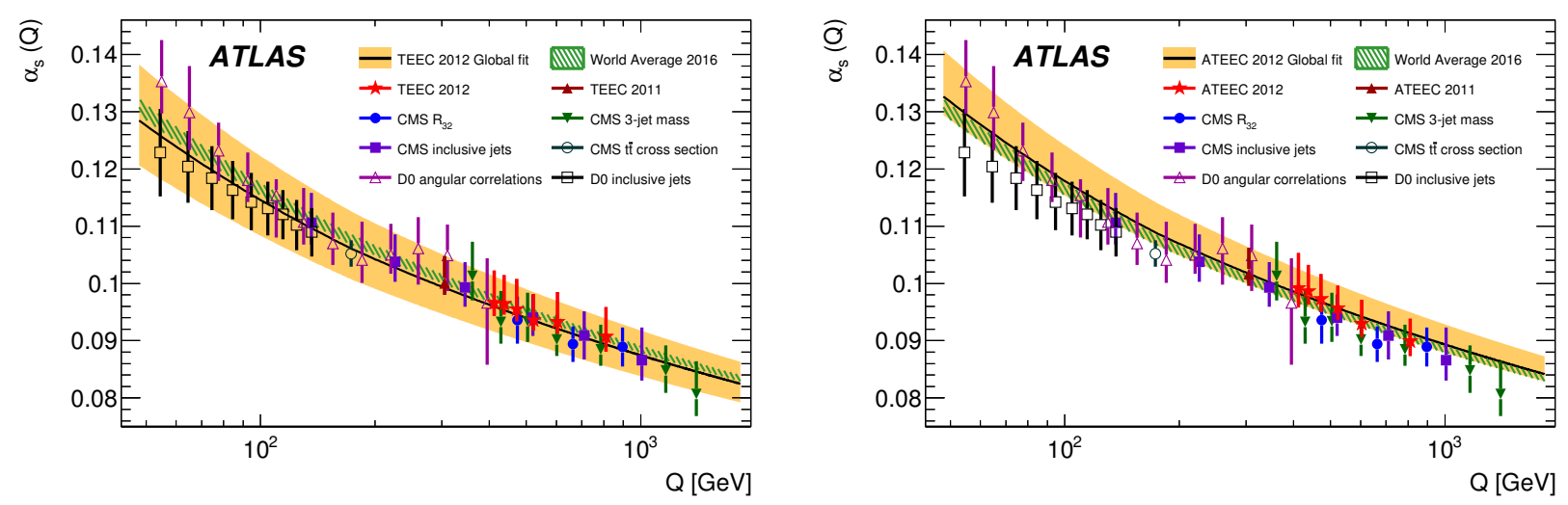

Figure 2: Scale dependence of $\alpha_{\mathrm{s}}$ values obtained from TEEC (left) and ATEEC (right) measurements [5].

\section{Measurement of azimuthal decorrelations}

The azimuthal decorrelations are defined as the fraction of the inclusive dijet cross-section for which the azimuthal difference between the two leading jets is smaller than a given value, $\Delta \phi_{\max },[8]:$

$$
R_{\Delta \phi}\left(H_{\mathrm{T}}, y^{*}, \Delta \phi_{\max }\right)=\frac{d^{2} \sigma_{\text {dijet }}\left(\Delta \phi_{\text {dijet }}<\Delta \phi_{\max }\right) / d H_{\mathrm{T}} d y^{*}}{d^{2} \sigma_{\text {dijet }}(\text { inclusive }) / d H_{\mathrm{T}} d y^{*}} .
$$

ATLAS has recently presented measurements on azimuthal decorrelations [9] as an alternative method to determine the strong coupling constant and to probe $\mathrm{pQCD}$ at high scales. Anti$k_{\mathrm{T}}$ jets $(\mathrm{R}=0.6)$ are selected with $p_{\mathrm{Tmin}}=100 \mathrm{GeV}$ and $|y| \leq 2.5$. The selection criteria require $H_{\mathrm{T}}=\sum_{i} p_{\mathrm{Ti}} \geq 450 \mathrm{GeV}, p_{\mathrm{T} 1}>H_{\mathrm{T}} / 3, y^{*}=\left|y_{1}-y_{2}\right| / 2<2$ and $\left|y_{i}-y_{\text {boost }}\right|<0.5$ with 
$y_{\text {boost }}=\left|y_{1}+y_{2}\right| / 2$. The data are presented as a function of $H_{\mathrm{T}}$ in three $y^{*}$ bins and four values of $\Delta \phi_{\max }$. The data are further corrected for detector effects using a bin-by-bin reweighting procedure. The systematic uncertainties are well under control, typically around the few percent level. The theoretical calculations have been performed as in the previous section using NLOJET++. The renormalization and factorization scales are set at $\mu_{\mathrm{R}}=\mu_{\mathrm{F}}=H_{\mathrm{T}} / 2$. The corrected data are shown in the left hand side of Fig. 3 along with a comparison to the NLO predictions. The agreement is
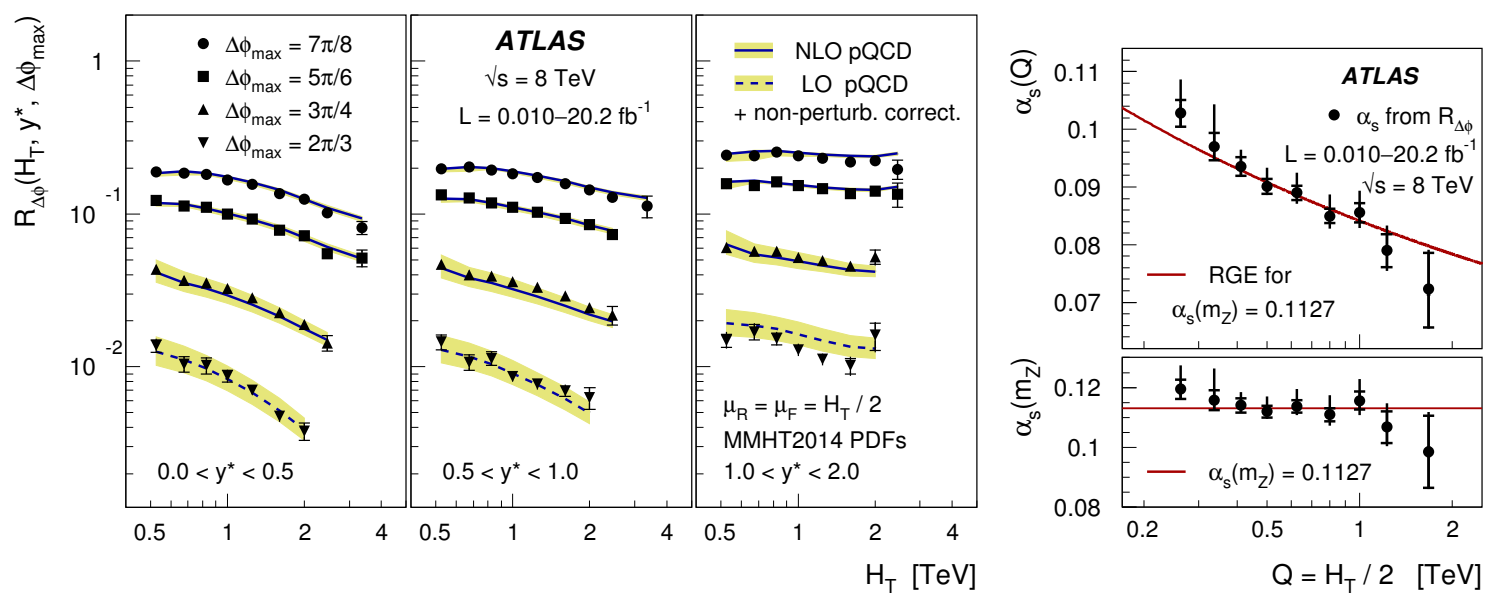

Figure 3: Left: Azimuthal decorrelations as a function of $H_{\mathrm{T}}$ compared to NLO pQCD predictions [9]. Right: Values of $\alpha_{\mathrm{s}}$ obtained from fits to $R_{\Delta \phi}$ for $\Delta \phi_{\max }=7 \pi / 8$.

fair. A closer look at the data/theory ratios indicates that the predictions do best for $\Delta \phi_{\max }=7 \pi / 8$ as expected. Therefore for a determination of $\alpha_{\mathrm{s}}$ the data for this particular value of $\Delta \phi_{\max }$ is integrated over $y^{*}$ and its $H_{\mathrm{T}}$ dependence fitted to pQCD predictions at NLO accuracy. The results of this fit are shown on the right hand side of Fig. 3. They yield the following value for the strong coupling constant at the $Z$ mass: $\alpha_{\mathrm{s}}^{\text {decorr }}\left(m_{\mathrm{Z}}\right)=0.1127_{-0.0027}^{+0.0063}$ (total). The total uncertainty is dominated again by the theoretical scales dependence.

\section{Summary and conclusions}

To summarize, two recent results on jet physics from the ATLAS collaboration at the LHC have been discussed as ways to probe $\mathrm{pQCD}$ at high scales. The main quantitative result is that the coupling constant has been measured with good precision as illustrated in Fig. 4 and its running tested to unprecedented scales of the order of $2 \mathrm{TeV}$. As a matter of fact, the level of experimental precision is similar to that of the LEP experiments. This calls for improved calculations beyond the NLO accuracy for three jet cross sections in pp collisions [10].

Copyright 2018 CERN for the benefit of the ATLAS Collaboration. Reproduction of this article or parts of it is allowed as specified in the CC-BY-4.0 license. 

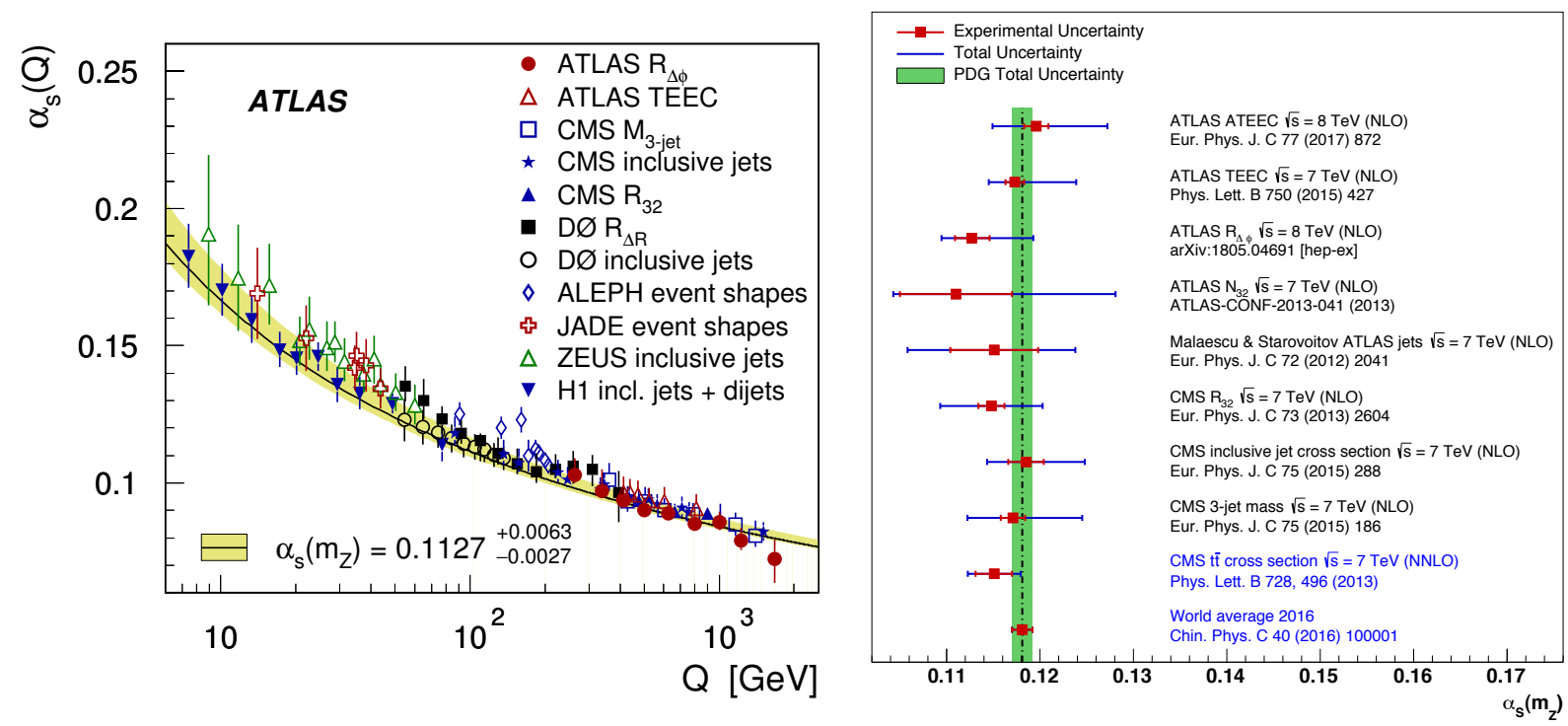

Figure 4: Left: Dependence of $\alpha_{\mathrm{s}}$ on the scale from [9]. Right: Summary of $\alpha_{\mathrm{s}}\left(m_{\mathrm{Z}}\right)$ values obtained at colliders. In blue, those based on NNLO calculations. In green is the PDG average value [11].

\section{References}

[1] ATLAS Collaboration, JINST 03 (2008) S08003.

[2] A. Ali, E. Pietarinen and J. Stirling, Phys. Lett. B 141 (1984) 447.

[3] A. Ali, F. Barreiro, J. Llorente and W. Wang, Phys. Rev. D 86 (2012) 114017.

[4] Z. Nagy, Phys. Rev. D 68 (2003) 094002.

[5] ATLAS Collaboration, Eur. Phys. J. C 77 (2017) 892.

[6] ATLAS Collaboration, Phys. Lett. B 750 (2015) 427.

[7] J. Llorente and B. P. Nachman, Nucl. Phys. B 936 (2018) 106 [arXiv:1807.00894 [hep-ph]].

[8] M. Wobisch and K. Rabbertz, JHEP 12 (2015) 024.

[9] ATLAS Collaboration, Phys. Rev. D 98 (2018) 092004.

[10] A. Gao, H. T. Li, I. Moult and H. X. Zhu, arXiv:1901.04497 [hep-ph].

[11] M. Tanabashi et al. [Particle Data Group], Phys. Rev. D 98 (2018) 030001. 